\title{
BUCHBESPRECHUNGEN
}

\section{Edda Blenk-Knocke}

$\mathrm{Zu}$ den soziologischen Bedingungen völkerrechtlicher Normenbefolgung Die Kommunikation von Normen

Münchener Universitätsschriften - Abhandlungen zur rechtswissenschaftlichen Grundlagenforschung Bd. 40, Verlag Rolf Gremer, Ebelsbach 1979, XII, 231 S.

Die Verbindung von Völkerrecht und Rechtsoziologie, die der Titel des besprochenen Buches anzeigt, verheißt zumindest dann wissenschaftliches Neuland, wenn es um mehr geht, als eine originelle Terminologie. Angesichts der eher konventional als logisch begründeten sozialwissenschaftlichen Fächereinteilung spricht nämlich nichts Grundlegendes dagegen, die (politikwissenschaftliche) Lehre von den Internationalen Beziehungen als "Soziologie" des Völkerrechts zu bezeichnen, wie es kein Geringerer als Max Huber schon 1910 getan hat ${ }^{1}$, und daß sozialwissenschaftliche Erkenntnisse generell Relevanz für das Völkerrecht haben, dürfte kaum noch bezweif elt werden, auch wenn nicht immer die Konsequenzen aus dieser Erkenntnis gezogen werden. Tatsächlich geht es der Verfasserin u. a. auch um eine neue Terminologie. Sie möchte die sozialwissenschaftlichen Grundlagen des Völkerrechts in einem inderdisziplinären (aus Internationalen Beziehungen, Völkerrecht und Rechtssoziologie gespeisten) Fach "Völkerrechtssoziologie" zusammenfassen (S. 33 ff.). Dahinter steht die richtige Erkenntnis, daß für eine sozialwissenschaftliche Behandlung des Rechts auf die traditionellen sozialwissenschaftlichen Fächergrenzen keine Rücksicht genommen werden kann, sondern jeweils entsprechend der rechtlichen Fragestellung fächerübergreifend gearbeitet werden muß. Ob der Ausdruck "Völkerrechtssoziologie" diesen Sachverhalt besonders glücklich bezeichnet, mag angesichts des deutlichen Úbergewichts der Internationalen Beziehungen in der anvisierten "Fächermischung" bezweif elt werden, doch kann die terminologische Frage dahingestellt bleiben, da die Verfasserin ihre Terminologie nicht zur bloßen Umettiketierung benutzt, sondern ihr Buch tatsächlich auf die Bedeutung der Rechtssoziologie "im engeren Sinne" für das Völkerrecht konzentriert und damit in der Tat wissenschaftliches Neuland betritt.

Das geschieht zum einen durch die Befragung soziologischer Rechtstheorien auf ihre Anwendbarkeit auf das Völkerrecht (S. 41 ff.) - eine Untersuchung, die durchgehend ergibt, daß vom Rechtsbegriff dieser Theorien her dem Völkerrecht schwer, wenn überhaupt beizukommen ist (ein Ergebnis, daß natürgemäß sowohl gegen die Allgemeingültigkeit der jeweiligen Definition wie auch gegen die Rechtsnatur des Völkerrechts sprechen kann).

Hauptthema ist dann das klassische rechtssoziologische Thema der "Effektivität" von Recht, und unter den Effektivitätsbedingungen die Kommunikation von Normen. Wäre man durch den Untertitel der Arbeit nicht auf diese Einschränkung vorbereitet, käme sie 
eher überraschend, denn die materialreichen Ausführungen der Verfasserin über die sozialwissenschaftlichen Charakteristika des Völkerrechts (S. 64 ff.) und dessen Wirksamkeitsvoraussetzungen (S. 86 ff.) legen kaum den Schluß nahe, daß die Kommunikation von Normen, zumal die an Private, der ein Großteil der weiteren Ausführungen gewidmet sind (S. 144 ff.), das zentrale Problem einer "Völkerrechtssoziologie" sind. Es ist durchaus fraglich, ob die Bedeutung der Normkommunikation nicht auch in der allgemeinen rechtssoziologischen Effektivitätsforschung überschätzt wird, das Völkerrecht erscheint jedenfalls als Musterbeispiel eines Rechtsbereiches, in dem es viel weniger auf die Normkenntnis der beteiligten Akteure als vielmehr auf ihre Fähigkeit und Bereitschaft, die Normen zu befolgen, und - noch grundlegender - auf ihre Einigkeit über den Inhalt der Normen ankommt.

Das soll nicht heißen, daß Völkerrechtskenntnisse der breiten Offentlichkeit wegen einer möglichen Rückkoppelung auf das Verhalten staatlicher Akteure völlig irrelevant sind, und die empirische Untersuchungen der Verfasserin zur Ubermittlung völkerrechtlicher Kenntnisse im schulischen und außerschulischen Bildungsbereich (S. 153 ff., mit dem zu erwartenden Negativbefund) sind zweifellos interessant. Nur erscheinen diese Randthemen als wenig geeignete Beispiele für die Notwendigkeit und Fruchtbarkeit einer Verbindung von Völkerrecht und Rechtssoziologie, die die Verfasserin so überzeugend vertritt.

Trotz dieser Kritik bleibt der Wert (und die Pionierleistung) des Buches mit seiner breiten Bestandsaufnahme der Beziehungspunkte zwischen Völkerrecht und Rechtssoziologie (wie auch der Forschungsdefizite) unbestreitbar.

Brun-Otto Bryde

\author{
W. Poeggel R. Meißner Ch. Poeggel \\ Staatennachfolge in Verträge \\ Staatsvertrag der Deutschen Demokratischen Republik (Ost-)Berlin 1980, 211 S.
}

Das Recht der Staatennachfolge, eines der schwierigsten und umstrittensten Gebiete des Völkerrechts, gehörte schon seit 1949 zu den Kodifikationsvorhaben der Völkerrechtskommission der Vereinten Nationen (ILC). Nach 16-jähriger Arbeit ist es 1978 gelunden, mit der Unterzeichnung der "Wiener Konvention über das Recht der Staatennachfolge in Verträge" (WKSV) ein erstes Teilgebiet der beabsichtigten Kodifikation abzuschließen. Die Normierung der Nachfolge in Rechte und Pflichten aus anderen Quellen als Verträgen ist dagegen noch nicht vollendet, während die Nachfolge in die Mitgliedschaft internationaler Organisationen vorläufig von einer Behandlung durch die ILC ausgespart worden ist. Da die Konvention über die Nachfolge in Verträge jedoch nach ihrem Art. 49 erst dann in Kraft treten kann, wenn mindestens 15 Staaten die Ratifikation oder den Beitritt erklärt haben, bleibt abzuwarten, ob ihr nicht dasselbe (zumindest vorläufige) Schicksal zuteil wird wie der Wiener Vertragskonvention von 1969, die bis 